\begin{tabular}{|l|l} 
REVISTA Revista Educación \\
ISSN: 0379-7082 \\
ISSN: $2215-2644$ \\
revedu@gmail.com \\
Universidad de Costa Rica \\
Costa Rica
\end{tabular}

\title{
Sentidos y significados de la formación ciudadana en tres escuelas colombianas
}

\author{
Lara Salcedo, Luz Marina \\ Sentidos y significados de la formación ciudadana en tres escuelas colombianas \\ Revista Educación, vol. 45, núm. 2, 2021 \\ Universidad de Costa Rica, Costa Rica \\ Disponible en: https://www.redalyc.org/articulo.oa?id=44066178036 \\ DOI: https://doi.org/10.15517/revedu.v45i1.42799
}

\section{(c) $(1) \Theta(9$}

Esta obra está bajo una Licencia Creative Commons Atribución-NoComercial-SinDerivar 3.0 Internacional. 


\section{Sentidos y significados de la formación ciudadana en tres escuelas colombianas}

Sense and Meaning of Citizenship Training at Three Colombian schools

Luz Marina Lara Salcedo

Pontificia Universidad Javeriana, Colombia

DOI: https://doi.org/10.15517/revedu.v45i1.42799

laral@javeriana.edu.co

Redalyc: https://www.redalyc.org/articulo.oa?

(DD https://orcid.org/0000-0003-0182-6878 $\mathrm{id}=44066178036$

Recepción: 15 Julio 2020

Aprobación: 06 Noviembre 2020

\section{Resumen:}

El artículo se deriva de una investigación cuyo objetivo fue comprender la configuración de prácticas educativas en formación ciudadana. El enfoque del estudio fue descriptivo-comprensivo, ya que se pretendía describir los rasgos de tres experiencias significativas para comprender sus sentidos y significados. La perspectiva teórica de análisis fue el socioconstruccionismo. El diseño metodológico fue estudio de caso y, como técnicas de recolección de datos, se aplicaron entrevistas a profundidad al personal docente líder de las experiencias; semiestructuradas al personal rector, coordinador, al profesorado y a las madres y los padres del estudiantado, y grupos focales al estudiantado. Los datos se procesaron mediante el Análisis de Contenido. Los hallazgos permitieron comprender que las experiencias movilizan un trabajo interdisciplinario, que la formación política del estudiantado es la principal preocupación del personal docente, y confirmaron procesos formativos que transitan de una educación cívica con enfoque en la civilidad hacia una formación ciudadana que promueve la participación y la toma de decisiones. Sin embargo, es necesario fortalecer las redes del profesorado para intercambiar experiencias y conocimientos que enriquezcan la discusión y el debate en la formación ciudadana, para formar semilleros de investigación orientados a la formación ciudadana del estudiantado.

Palabras Clave: Formación política, Ciudadanía, Educación ciudadana, Escuelas, Colombia.

\section{Abstract:}

A descriptive-comprehensive study was conducted to better understand citizenship training teaching practices. The study sought to examine characteristics of three significant case studies to probe into their sense and meaning using a socio-constructionism theoretical perspective. Data collection techniques were based on in-depth interviews conducted among leading teachers, and semi-structured principals, coordinators, teachers and parents; focus groups were held with the students and data processed through content analysis. Based on the findings, we were able to understand that experiences foster interdisciplinary work and that teachers are mainly concerned with the political development of their students and developing lessons on citizenship education that fosters student engagement in decision-making. However, teachers must strengthen their networks and share best practices and experiences to enrich the discussion and the debate in citizenship training as well as identify research for citizenship training. KEYWORDS: Political Education, Nationality, Citizenship Education, Schools, Colombia.

\section{ANTECEDENTES}

En el año 2012, en el marco del Programa de Competencias Ciudadanas, el Ministerio de Educación Nacional de Colombia (MEN) y el Comité Internacional para el Desarrollo de los Pueblos (Comitato Internazionale per lo Sviluppo dei Popoli [CISP]) llevaron a cabo un proceso de sistematización de quince experiencias significativas del país en competencias ciudadanas, con el fin de visibilizarlas y lograr su institucionalización o inclusión en los Proyectos Educativos Institucionales [PEI], para garantizar su reconocimiento legal e implementación para el desarrollo de las competencias que el estudiantado requiere para una ciudadanía activa.

En este contexto, y con el propósito de profundizar en la sistematización, surgió la presente investigación, cuyo objetivo fue dar un paso más allá para comprender los ámbitos de preocupación de las personas maestras en torno a sus prácticas formativas en ciudadanía, las estrategias de enseñanza privilegiadas, las 
intencionalidades sociales, políticas que perseguían, y las resignificaciones elaboradas en clave de la formación ciudadana del estudiantado. A continuación, se presentan los hallazgos de tres experiencias significativas en tres instituciones educativas colombianas, ubicadas en las ciudades de Quibdó, Bogotá y Barranquilla. El artículo se estructura en cinco apartados: antecedentes, referentes teóricos que orientaron el trabajo investigativo, horizonte metodológico, análisis de resultados y discusión, y principales conclusiones del estudio.

Las investigaciones que buscaban identificar los rasgos característicos de las prácticas en formación ciudadana, no eran muchas en ese entonces. Un primer ejercicio se realizó en el Programa de Experiencias Significativas de la Subdirección de Fomento de Competencias del Ministerio de Educación Nacional [MEN], que derivó en el año 2008 en la ruta de acompañamiento, conocida como Rutas del Saber Hacer, que reúne información de una gran cantidad de experiencias que se sistematizaron en el año 2012. Entre los hallazgos arrojados por esta sistematización, se resaltaba que las competencias ciudadanas eran aprovechadas para abordar problemas del contexto social, así como dificultades en relación con el bajo rendimiento académico del estudiantado, motivación hacia las clases y el fomento de las competencias cognitivas, comunicativas y emocionales (MEN y CISP, 2012). Asimismo, se encontraron importantes logros en el desarrollo de las competencias conocidas como integradoras para solucionar problemas, que demandaban por parte del estudiantado tener competencia cognitiva, comunicativa y emocional.

Por otro lado, el Informe Regional 2010. Evaluación de Programas Exitosos de Formación Ciudadana (Pontificia Universidad Católica de Chile, 2010) evaluó 67 programas en 6 países de América Latina (Chile, Guatemala, Colombia, México, República Dominicana y Paraguay), esto con el propósito de comparar los diseños de los programas junto con su puesta en marcha a partir de 4 categorías: objetivos, metodología, gestión y resultados. En este se señalaba que la mayoría de los trabajos realizados enfatizaban en el desarrollo de actitudes y habilidades, con poco interés por el desarrollo de conocimientos; que contaban con una buena fundamentación teórica y sus metodologías participativas eran acordes con sus objetivos. Entre sus debilidades se señalaba la falta de una evaluación sistemática de los programas y escasos recursos económicos y de tiempo para su implementación.

En el mismo año, la Fundación Centro Internacional de Educación y Desarrollo Humano [CINDE] realizó un estudio de 44 programas nacionales en formación de competencias ciudadanas, financiados por el MEN e implementados en conjunto con las Secretarías de Educación, Escuelas Normales Rurales, entidades gubernamentales, organizaciones no gubernamentales, empresas privadas, instituciones universitarias y organismos internacionales. Algunos hallazgos revelaban que las áreas curriculares de mayor desarrollo eran: Ética, Valores Humanos, Ciencias Sociales y, en menor medida, Lenguaje, Ciencias Naturales, Constitución Política y Filosofía.

En este estudio, los programas enfatizaban en la promoción de la convivencia, el gobierno escolar, la justicia escolar y su gestión desde la institución. Además, se encontró que promovían la participación del estudiantado a través de la toma de decisiones, la aceptación activa de las diferencias, el manejo del conflicto y el desarrollo de habilidades argumentativas (CINDE, 2010). Adicionalmente, se resaltaba que la dimensión más abordada era la cognoscitiva, lo que fortalecía el desarrollo del pensamiento crítico y reflexivo para aprender a convivir; el conocimiento sobre cómo funciona el Estado, y el empoderamiento de los derechos. A esta le seguía el área socioemocional, relacionada con el ejercicio ciudadano. También, se resaltaba que los sentimientos eran asumidos como habilidades para interactuar y establecer relaciones de convivencia, que favorecían conductas proactivas como la empatía, la asertividad y el desarrollo de habilidades de inteligencia emocional (CINDE, 2010). Entre las principales debilidades se encontró que un escaso número de programas promovían la deliberación y que la especificidad de lo ciudadano no era claramente visible.

Ahora bien, el estado del arte sobre formación ciudadana en Colombia 1994-2004, llevado a cabo por Pinilla Díaz y Torres Azocar (2006), evidenciaba la carencia de un núcleo articulador en torno a las dimensiones de la formación ciudadana (educación cívica, democracia, cultura política, socialización política, 
entre otros) y que no existía una síntesis teórica que abordara claramente las categorías de ciudadanía, formación ciudadana y ciudadanía escolar. Por lo tanto, se evidenciaba una falta de reflexión teórica y un exceso de actividades con el estudiantado en torno a la ciudadanía.

En Desafios de la formación ciudadana en la era digital: Estado del arte, Silva y Tapia Zúñiga (2015) señalan que, en el ámbito latinoamericano y desde una filosofía crítica, las concepciones actuales de ciudadanía estaban vinculadas al enfoque republicano, se buscaba superar los paradigmas liberales y comunitarios. Esto con el fin de poner en un mismo plano la autonomía privada y pública, para configurar una democracia deliberativa donde todas las personas participaran. De acuerdo con los autores, "De esta manera hace su aparición conceptualmente el ciudadano activo y participativo, contrario al ciudadano que solo participa (de manera pasiva) en las elecciones" (Silva y Tapia Zúñiga, 2015, p. 22).

Así, se ponía de relieve que la literatura especializada en formación ciudadana diferenciaba cuatro modelos de construcción. El primero orientado hacia la educación cívica; el segundo, hacia un conocimiento de lo político; el tercero, por una búsqueda reflexiva de los conocimientos asociados a la formación ciudadana, y el cuarto pretendía desarrollar la autonomía. Los dos primeros modelos respondían a lo que tradicionalmente se conocía como educación cívica y los dos últimos como formación ciudadana, la cual se incorporaría a la formación por competencias del MEN desde el enfoque de la ciudadanía activa, en el cual aprender y hacer eran inseparables.

En cuanto a los estudios que se centran en la institución educativa como eje de la formación ciudadana, diversos autores señalan que se requiere de su incorporación al currículo para garantizar el desarrollo de capacidades ciudadanas (Ñ́ñez Rodríguez y Castro Turriago, 2016; Conejo Rodríguez, 2012; Vargas Torres, 2010; Eidhof, ten Dam, Dijkstra y Van de Werfhorst, 2016; Caicedo Caviedes, 2015). Entonces, debería articularse con un área del conocimiento, en particular: Ciencias Sociales, Ética y Filosofía, o trabajarla como un componente transversal del currículo (Garzón Guerra y Acuña Beltrán, 2016).

Por otro lado, estudios como el de Muñoz Labraña y Torres Durán (2014) examinan los problemas y desafíos de la formación ciudadana para las instituciones educativas. $\mathrm{Al}$ respecto, tres estudios en particular bordean nuevos retos para las ciudadanías y la formación ciudadana en la actualidad. Estos son: el de Bickmore, Salehin Kaderi y Guerra Sua (2017), relacionado con las violencias actuales y la ciudadanía; el de de Oliveira Andreotti y Pashby (2013), que aborda la formación ciudadana a través de medios digitales y, finalmente, el de de Oliveira Andreotti (2011), en el que se analizan las ciudadanías globales.

\section{REFERENTES TEÓRICOS}

\section{Socioconstruccionismo}

La perspectiva teórica que orientó esta investigación a nivel epistemológico fue el Socioconstruccionismo, la cual permitía comprender el constructo social formación ciudadana desde la experiencia del personal docente, sus pautas de relación y el lenguaje utilizado en ellas, al reconocer que por medio del lenguaje se construyen realidades. Desde esta perspectiva se plantea que el conocimiento no está en la mente de las personas, ni se deriva de una naturaleza preexistente, y que las palabras no son reflejo de esta. Así,

la fuente principal de las palabras que utilizamos sobre el mundo radica en la relación social. Desde este ángulo, lo que llamamos conocimiento no es el producto de mentes individuales, sino del intercambio social; no es el fruto de la individualidad sino el de la interdependencia (Gergen, 1982, p.169).

Entonces, se trata de una epistemología social, en la cual "el lugar del conocimiento ya no es la mente del individuo, sino más bien las pautas de relación social” (Gergen, 1999, p. 167). Una de sus premisas centrales es que las formas en las que se da cuenta de las comprensiones del mundo están determinadas por la cultura, la historia y el contexto social (Gergen, 1982; 1999). En esta misma línea, se plantea que las concepciones del 
mundo social, e incluso acerca de las personas, son artefactos sociales construidos cultural e históricamente a partir del lenguaje. De esta manera, tanto las pautas de relación como el lenguaje construyen realidades y las transforman, con lo que se constituye este lugar epistemológico como el punto de ubicación del objeto de estudio: la formación ciudadana como un constructo social y cultural. En este sentido, de acuerdo con Gimeno Sacristán (2001), se concibe la ciudadanía como una forma de ejercer la socialidad en el seno de la sociedad jurídicamente regulada, que garantiza a los individuos el ejercicio de la igualdad, la libertad, la autonomía y los derechos de participación.

\section{Experiencias significativas}

En esta investigación se adoptó el concepto de experiencia significativa planteado por el Ministerio de Educación Nacional de Colombia [MEN], el cual hace referencia a una práctica de enseñanza que busca desarrollar aprendizajes significativos al desarrollar competencias en el estudiantado. Por consiguiente, se trata de una práctica que busca respuestas innovadoras frente a las necesidades académicas o sociales, que está fundamentada teóricamente, es coherente en términos metodológicos y produce impactos dentro y fuera de la escuela. De este modo, se aporta al fortalecimiento de los componentes: académico, directivo, administrativo y comunitario en los establecimientos educativos, lo que redunda en su calidad educativa. De acuerdo con el MEN (2010), una experiencia significativa está compuesta por las siguientes características:

- Tiene varios años de implementación, lo que le otorga madurez.

- Es sistemática, es decir que sus acciones llevan un orden lógico.

- El logro de sus objetivos es evidente.

- Son autorreguladas, ya que analizan sus fortalezas y oportunidades de mejora.

- Planean sus actividades de acuerdo con el contexto y las necesidades de la comunidad educativa.

- Generan cambios en la cultura institucional y aportan soluciones innovadoras a las necesidades del estudiantado y del establecimiento educativo.

- Representan la consolidación de una práctica o de un modelo educativo en la institución educativa.

\section{CiUDADANÍA Y FORMACIÓN PARA LA CIUDADANÍA}

La ciudadanía es uno de los conceptos que más definiciones ha resistido a lo largo de la historia[1]. Como lo señala Zapata Borrero (2001), "el mayor problema del término ciudadanía, es que carece de concepto, tan sólo podemos hablar de concepciones" (p. 6). En términos generales, este expresa las relaciones entre el Estado y los miembros de una sociedad, que se constituyen como el criterio estatal que define quiénes son miembros y quiénes no.

Esta noción excluyente cambia con la modernidad europea, donde se fundamenta solo en dos criterios: nacionalidad e igualdad, los cuales aluden, según Zapata-Borrero (2001), a la pertenencia al mismo Estadonación y a la consideración como iguales de las personas que pertenecen a este, ya que gozan de los mismos derechos. Por su parte, Marshall (1950) formula la tesis de la expansión del concepto en las sociedades modernas e identifica la ciudadanía civil, política y social como ciudadanías que se basan en los Derechos Humanos.

Además, por su directa relación con el conocimiento de las leyes de gobierno, la ciudadanía se ha relacionado con la educación cívica. De acuerdo con Abowitz y Harnish (2006), la ciudadanía como aprendizaje es clave para la adquisición de conocimientos sobre el gobierno (constitución política, ciencia política, teorías de la democracia, etc.), ya que constituye lo que se considera como aprendizaje cívico o adquisición de saberes y disposiciones necesarias para ejercer la ciudadanía. En el contexto educativo colombiano, el modelo formativo, inicialmente, estuvo orientado hacia la educación cívica y la urbanidad, 
y buscaba educar en normas de buena conducta y comportamiento social para mantener unos principios básicos de convivencia. Sin embargo, este modelo se ha transformado en formación ciudadana, ya que su objetivo de formación pretende generar el empoderamiento en torno a los derechos, deberes y su aplicación en la dinámica del contexto social y político. De esta manera, se pasa de la educación cívica a la formación ciudadana, donde el ser ciudadano comienza a tener una nueva connotación: poseer valores, actitudes y comportamientos democráticos asociados a la libertad, el reconocimiento de la pluralidad, la participación, los referentes de identidad y la crítica argumentada.

En cuanto al currículo de la educación para la ciudadanía, Selwyn (2004) establece tres enfoques. En primer lugar, la educación sobre la ciudadanía, referida, como lo señala Marchesi (2011), a la reflexión para el desarrollo del juicio y la autonomía moral, articulados con la educación de los sentimientos y las emociones morales como la culpa, el altruismo, la empatía, la indignación y la vergüenza, "para evitar hacer daño a los otros o para solidarizarse con ellos y ayudarles" (p. 14). Así, se trasciende la adquisición de conocimientos sobre la historia nacional, el gobierno y sus estructuras, y la vida política. En segundo lugar, la educación por medio de la ciudadanía, orientada a que el estudiantado aprenda los valores cívicos desde su ejercicio en la escuela y la sociedad, y hacer un estilo de vida de la comunidad democrática en el ámbito escolar, caracterizado por la tolerancia, el respeto, la solidaridad y la responsabilidad hacia los demás. Finalmente, la educación para la ciudadanía, que comprende las anteriores: adquisición de conocimientos y desarrollo de actitudes, valores y habilidades para participar activamente y con responsabilidad en la construcción de sociedades más democráticas.

En este último enfoque se puede ubicar la formación para la ciudadanía mediante el desarrollo de competencias, que en el caso colombiano "son el conjunto de conocimientos y de habilidades cognitivas, emocionales y comunicativas que, articulados entre sí, hacen posible que el ciudadano actúe de manera constructiva en la sociedad democrática" (MEN, 2004, p. 8). Estas competencias aluden a habilidades cognitivas, emocionales, comunicativas e integradoras, y a unos conocimientos que facilitan la comprensión de la competencia para su implementación por parte del estudiantado, el cual se encuentra formulado y analizado en los Estándares Básicos de Competencias Ciudadanas del MEN (2003), y en publicaciones como las de Chaux, Lleras y Velásquez (2004); Rodríguez (2010); y Chaux (2012).

Desde una mirada más actual sobre las competencias ciudadanas, León Muñoz y López Takegami (2015) plantean que su abordaje en los primeros años de la educación inicial y primaria debe basarse en acciones ciudadanas relacionadas con el respeto a la diferencia y del otro, la solidaridad, el cumplimiento del deber, la resolución de conflictos, la convivencia pacífica, la pluralidad, la valoración y la participación ciudadana. Por su parte, López, Arán Filippetti y Richaud (2013) resaltan la importancia de trabajar la competencia emocional de la empatía en la formación ciudadana, para que el estudiantado aprenda a reconocer y comprender los estados afectivos de las personas y responder a ellos adecuadamente.

Sin embargo, desde una perspectiva crítica, para pensadores como Guillermo Hoyos Vásquez, Serna Arango y Gutiérrez Ruiz (2007), esta forma de concebir la formación ciudadana despoja a los ciudadanos de las posibilidades de construirse a sí mismos y del derecho de decidir libremente el tipo de persona que quieren ser y el mundo con el que sueñan. Se coincide con Hoyos Vásquez, Serna Arango y Gutiérrez Ruiz (2007) en abogar por una educación para el humanismo que, como proceso comunicativo, le apueste a la formación de ciudadanos y ciudadanas que sepan ejercer sus derechos, cumplir sus deberes y participar políticamente en la construcción de país. Precisamente este sentido ético-político fue el que se encontró en las experiencias estudiadas.

Ahora bien, planteamientos más recientes, como los de Avendaño Castro, Paz Montes y Parada Trujillo (2016), reconocen que la ciudadanía es, también, la construcción de escenarios permanentes en los que se pueda convivir con los otros, donde se construya una vida digna de ser vivida en la diversidad, es decir, con dignidad humana. Además, Saldarriaga Vélez (2016) la reconoce no solo como un asunto legal o de pertenencia territorial, sino como la construcción de un sujeto político desde la reflexión y la acción, lo cual se 
evidenció en las experiencias analizadas, y se constituye como la base de las tendencias actuales en formación ciudadana en el país.

\section{HoRIZONTE METODOLÓGICO}

El análisis, realizado desde la perspectiva del socioconstruccionismo, se dirigió a las construcciones que hicieron las personas informantes sobre sus experiencias. Por consiguiente, se definió como metodología el estudio de caso, que, de acuerdo con Sosa Cabrera (2002), es una investigación en profundidad para analizar el contexto y los procesos implicados en el objeto de estudio. Por lo tanto y de acuerdo con la autora, se le consideró como un estudio intensivo de ejemplos seleccionados, en los que el fenómeno no se aisló de su contexto.

El enfoque del estudio se orientó desde una perspectiva cualitativa, para establecer los rasgos, cualidades y dimensiones que configuran las experiencias como un caso. Por otro lado, la investigación acudió a dos tipos de fuentes. En primer lugar, fuentes documentales (documentos e informes acerca de cada una de las experiencias) y fuentes secundarias. En segundo lugar, a partir del trabajo in situ, consultó las fuentes primarias de los casos objeto de estudio.

\section{Participantes y casos}

Se consideran tres experiencias significativas en formación ciudadana de educación básica y media colombiana de tres instituciones educativas, ubicadas en las ciudades de Quibdó, Bogotá y Barranquilla. Participaron 3 docentes líderes, 9 docentes colegas (tres por experiencia), 24 estudiantes ( 8 por experiencia), 6 directivos ( 2 por institución educativa) y 6 madres y padres de familia ( 2 por institución educativa).

\section{Selección de los casos}

Para la selección de las tres experiencias sistematizadas por el Ministerio de Educación y con las cuales se configuró el caso, se tuvo en cuenta los siguientes criterios:

- Contar con mínimo 5 años de trayectoria.

- Formar parte del Proyecto Educativo Institucional [PEI].

- Responder a necesidades o problemáticas institucionales o del contexto.

- Demostrar evidencias de reflexión a partir de su sistematización.

- Haber sido socializadas en eventos académicos y tener publicaciones derivadas del proceso de sistematización.

- Haber generado impacto en el contexto escolar, comunitario, local y regional.

\section{Técnicas de recolección de información}

Se aplicó una entrevista en profundidad a cada docente líder para conocer el contexto de emergencia de la experiencia, su desarrollo y estado actual. Para explorar los ámbitos de preocupación del profesorado, los procesos de aprendizaje privilegiados, las intencionalidades sociales y políticas que perseguían, y las resignificaciones elaboradas en torno a la formación ciudadana del estudiantado, se realizó una entrevista semiestructurada al personal rector de cada institución educativa, al personal a cargo de la coordinación académica, a tres docentes con conocimiento de la experiencia y a dos madres y padres de familia por institución, para así establecer sus comprensiones acerca del trabajo adelantado por el docente titular; y un 
grupo focal en el que participaron 8 estudiantes por colegio. La estructura de las entrevistas se organizó por categorías, por ejemplo, en el caso del guion para el personal coordinador, las personas docentes conocedoras de la experiencia y las madres y los padres de familia, se incluyeron seis categorías de preguntas: antecedentes de la experiencia, articulación curricular, componente investigativo, estrategias pedagógicas, evaluación e impactos de la experiencia. La entrevista al docente líder se estructuró en nueve categorías de preguntas: contextos de implementación de la experiencia, estrategias pedagógicas, articulación curricular, componente investigativo, enfoques o corrientes pedagógicas que la fundamentan, intencionalidades ético-políticas, logros en formación ciudadana, resignificaciones en formación ciudadana, vinculación a redes y relaciones con el sector externo. El formato para el grupo focal con el estudiantado abordó las preguntas en cinco categorías: conocimiento de la experiencia, metodologías desarrolladas, participación, comprensiones sobre formación ciudadana, aportes e impactos en formación ciudadana.

También, se llevó a cabo un proceso de observación de aula, cuyos datos se registraron en una guía de observación semiestructurada que se analizó por medio de una matriz, cuyo eje central fue la práctica pedagógica en relación con la formación ciudadana.

\section{ANÁLISIS DE DATOS}

El procesamiento de los datos se hizo mediante el Análisis de contenido para las producciones textuales obtenidas con las fuentes primarias y secundarias, esto con el fin de hacer la reducción de datos, codificar las categorías deductivas (las cuales fueron: ámbitos de preocupación, procesos de aprendizaje privilegiados, estrategias, intencionalidades políticas y resignificaciones en la formación ciudadana), configurar subcategorías e identificar las inductivas o emergentes (Derechos Humanos, pedagogía urbana, identidad cultural, formación política y escuela-ciudad). Posteriormente, se procedió al análisis intracategorial e intercategorial. Para ello, los datos se dispusieron, inicialmente, en una matriz categorial por institución educativa y por informante; posteriormente, se procedió a comparar las experiencias de las tres instituciones educativas, con el fin de ponerlas a dialogar, identificar elementos transversales y lograr una mayor inteligibilidad de acuerdo con los informantes. Un ejemplo de matriz categorial se muestra a continuación en la Tabla 1: 
TABLA 1

Modelo de matriz categorial con subcategorías docente líder

\begin{tabular}{|c|c|}
\hline Categorías & Subcategorías \\
\hline \multirow{3}{*}{$\begin{array}{l}\text { 1. Antecedentes de la } \\
\text { experiencia. }\end{array}$} & 1.1 Contexto de emergencia. \\
\hline & 1.2 Problemas y preguntas. \\
\hline & 1.3 Objetivos. \\
\hline \multirow{3}{*}{$\begin{array}{l}\text { 2. Articulación } \\
\text { Curricular }\end{array}$} & 2.1 Con el currículo. \\
\hline & 2.2 Con el plan de estudios. \\
\hline & 2.3 Con otros proyectos. \\
\hline \multirow{4}{*}{$\begin{array}{l}\text { 3.Componente } \\
\text { investigativo }\end{array}$} & 3.1 Rol de los estudiantes. \\
\hline & 3.2 Rol de los docentes. \\
\hline & 3.3 Metodología. \\
\hline & 3.4 Productos derivados. \\
\hline \multirow{4}{*}{$\begin{array}{l}\text { 4. Enfoques o } \\
\text { corrientes pedagógicas }\end{array}$} & 4.1 Fundamentos teóricos. \\
\hline & 4.2 Cómo se reflejan. \\
\hline & 4.3 Justificación del enfoque. \\
\hline & 4.4 Productos derivados. \\
\hline \multirow{4}{*}{$\begin{array}{l}\text { 5. Intencionalidades } \\
\text { ético-políticas }\end{array}$} & 5.1 Concepción de lo ético en la experiencia. \\
\hline & 5.2 Concepción de lo político en la experiencia. \\
\hline & $\begin{array}{l}5.3 \text { Implicaciones ético-políticas de la } \\
\text { experiencia. }\end{array}$ \\
\hline & $\begin{array}{l}\text { 5. } 4 \text { Relaciones de esta dimensión con la } \\
\text { formación ciudadana. }\end{array}$ \\
\hline
\end{tabular}

Fuente: elaboración propia

Por otra parte, en el proceso de elaboración y análisis de los datos se acudió al diseño de tablas categoriales a tres voces, en las cuales se partió de la voz del informante, se acudió al referente teórico y, posteriormente, se incluyó la voz interpretativa del personal investigador, para pasar de un nivel superficial a un nivel más profundo e interpretativo (ver Tabla 2):

TABLA 2

Modelo de tabla analítica categorial a tres voces

\begin{tabular}{|l|l|l|}
\hline Categoría Formación política & \multicolumn{2}{|l|}{} \\
\hline Voz del informante & Voz del referente teórico & Voz del personal investigador \\
\hline Entendimos que un proyecto de & "La formación política es un & Pensarse políticamente fue un objetivo \\
derechos humanos en la escuela & requisito para el ejercicio & que trascendió el aula de clase para \\
es una propuesta de formación & efectivo de los derechos & convertirse en la razón de ser de la \\
política como tal, que desborda & humanos y de las libertades & formación ciudadana. De esta manera, \\
los límites que a veces impone lo & fundamentales, por lo tanto, & los elementos políticos brindaron la \\
pedagógico. Entonces, nosotros lo & conceder una atención & posibilidad de pensar la escuela como \\
concebimos desde sus inicios & particular a la educación en & escenario para la participación y la \\
como un proyecto de formación & derechos humanos y en & toma de decisiones. En este sentido, la \\
política, ya que la educación la & democracia es una & formación política del estudiantado \\
entendemos como un acto político & condición esencial para el & tomó fuerza en la medida en que se \\
y afectivo que da sentido a los & pleno desarrollo de la & abrió a la posibilidad de reflexionar en \\
Derechos Humanos en la escuela & justicia" Jares, 2006, p. 58). & su ejercicio participativo y no \\
(docente líder). & & \\
\hline
\end{tabular}

Fuente: elaboración propia

Finalmente, la triangulación de resultados se llevó a cabo en la ciudad de Bogotá con los docentes líderes en un simposio de presentación de resultados. 


\section{Resultados}

En cada una de las experiencias, el marco en el que se configuró la enseñanza de la formación ciudadana fue distinto. Así, en la primera se realizó a partir de la enseñanza de las Ciencias Sociales, en la segunda mediante un proyecto interdisciplinario relacionado con la formación en Derechos Humanos y en la tercera trabajando la pedagogía urbana en el área de Sociales.

\section{Enseñanza y aprendizaje de las Ciencias Sociales escolares: un asunto de ciudadanía}

La primera experiencia significativa, Historichoquiando: Andamos haciendo historia sobre Chocó y Quibdó, daba cuenta de cómo la enseñanza y el aprendizaje de las Ciencias Sociales había adquirido nuevas formas. De este modo, pretendía responder a los retos, tanto sociales y políticos como académicos y educativos, que tenían como último propósito formar para la ciudadanía.

\section{Sentidos y significados de la enseñanza de las Ciencias Sociales escolares y la Historia en la experiencia.}

Para resignificar los sentidos de la enseñanza del área se partió de la comprensión de que el pasado era una reconstrucción social y cultural, susceptible de ser elaborada por los sujetos y las comunidades. De esta manera, se respondía a las necesidades del contexto y, también, a las tendencias actuales de enseñanza de las Ciencias Sociales y la Historia, las cuales explicitaban que los objetivos del aprendizaje social estaban orientados, por una parte, a tener conocimientos que posibilitaran comprender el contexto y el presente, y, por otra, a formar la conciencia histórica para que los sujetos se constituyeran como agentes transformadores y constructores de sus propias realidades.

Adicionalmente, la participación democrática de las personas participantes era fundamental en la experiencia y, como lo indicaban Benejam y Pagés (2002, citado en González Valencia, Posada López y Valencia Calvo, 2012), las Ciencias Sociales contribuían a la formación para la ciudadanía cuando se realizaban prácticas que promovían el respeto de la dignidad, la cooperación, la valoración de los rasgos de identidad y el reconocimiento y promoción de su propia herencia cultural. En este aspecto, uno de los principales logros fue acercar al estudiantado a la comprensión de la historia y la memoria local, mientras ejercen la labor de historiadores e historiadoras y hacen uso de algunos de los métodos de indagación de esta disciplina. Por ejemplo, investigaron sobre mitos y leyendas, los primeros pobladores, la conservación de los recursos naturales y el origen de su riqueza cultural desde la voz de sus familiares.

(...) hemos aprendido a saber valorar y conocer esa cultura que nuestros antepasados nos han dejado, para que así nosotros [podamos] transmitírsela a las demás personas. Yo soy ciudadano cuando aprendo a ser persona respetuosa de lo público y de lo ajeno. Cuando respeto las culturas de los demás y las comparto, incluso, en eso nos ha contribuido Historichoquiando. (E.1, comunicación personal, marzo de 2014).

Como lo señalaban Carretero y Montanero (2008), el pensamiento histórico era mucho más que la acumulación de información, por lo tanto, la enseñanza que acercaba al estudiantado al método de la historia permitía su formación en la habilidad para valorar de manera crítica las fuentes y toda la información que se recolectaba, lo que ayudó a desarrollar un pensamiento crítico de tipo social.

Esto le permitía al estudiantado comprender que la construcción del conocimiento histórico no estaba exenta de interpretaciones ideológicas. Además, contribuía a la formación de su pensamiento histórico, y a su formación ciudadana, pues posibilitaba que no solo interpretaran su realidad, sino que tuvieran herramientas intelectuales para actuar sobre ella. 


\section{La experiencia museal: reconocimiento del patrimonio cultural y una estrategia de formación ciudadana.}

La experiencia utilizaba como método de conocimiento la investigación, por medio de ella el estudiantado se relacionaba de forma distinta con el saber. Por ejemplo, elaboraron un registro sobre cómo se fue poblando el territorio, por medio de mapas y maquetas realizadas por ellos y ellas, o a partir de cuentos y leyendas narrados por sus mayores y que, luego, escribieron. Debe mencionarse que la misma existencia del museo era la respuesta a la necesidad de reorganizar y recrear un espacio que cambiara la relación con el entorno, para la resignificación y reapropiación de un espacio subutilizado, pues el lugar de ubicación de este estaba destinado para botar los desechos. De esta manera, se dio respuesta, desde la experiencia, a hechos concretos de su realidad inmediata. Al respecto, una madre de familia señaló lo siguiente:

Bueno, en primer lugar, algo que yo he observado, que le ha dado como valor a este espacio, le ha dado valor a las personas que viven alrededor, a la comunidad. Porque, por ejemplo, el museo se llama Museo quebrada La playita. Dice la comunidad, 'tenemos un museo que tiene nuestro nombre', entonces, es una forma de hacer visible esa comunidad, ¿cómo?, la gente ya no va a decir 'este es el barrio... Por allá abajo... ¿Dónde?’ No, sino que 'allá, en ese barrio, tienen una institución, tienen un parque ecológico y tienen un museo', y la misma comunidad se involucró en la construcción de este proyecto. (P.1, comunicación personal, marzo de 2014)

Otros proyectos de investigación se centraron en la recuperación de la memoria colectiva para conocer su cosmovisión, rescatar aspectos culturales y conservar valores culturales de tipo comunitario, como la Minga o la manera en la que la comunidad se organizaba para tomar decisiones y realizar labores conjuntas.

Pues, primeramente, hicimos visitas a nuestros alrededores e hicimos reuniones con los profesores, compañeros, estudiantes y la comunidad que nos rodea. Hicimos una planeación de cómo íbamos a distribuir los equipos de trabajo, las preguntas, a quién íbamos a entrevistar y cómo lo íbamos a hacer, y qué íbamos a tener en cuenta de los criterios que más nos interesaba destacar de nuestra cultura. Además de esos equipos de trabajo, hicimos una especie de vestuario, unas camisetas que nos distinguieran como equipo de trabajo, como grupo de investigación de la Escuela Normal Superior de Quibdó. Acompañados de los profesores y de los padres de familia fuimos investigando, y eso fue una de las series de actividades que más nos llamó la atención y la respuesta que nos daban nuestros ancestros, ¿cierto? Las personas más adultas fueron a las que más les consultamos sobre estos temas. (E.2, comunicación personal, marzo de 2014)

Adicionalmente, el personal docente destacó que, por un lado, las investigaciones siempre tenían como producto objetos tangibles que se exponían para darlos a conocer en el museo. Por otro lado, que lo más valioso era la exposición final que hacía el estudiantado del proceso. Ello le permitía comprender y dar una explicación del porqué de su existencia. Entonces, esto se constituía como una dinámica que les posibilitaba generar conocimiento y comprensiones de su propia cultura.

Otro aspecto sobresaliente era que el método de investigación histórico utilizado desarrollaba en el estudiantado el pensamiento crítico, ya que la consulta para sus indagaciones era, principalmente, de tipo oral. Ello demandaba un trabajo de interpretación crítica y comprensión, ejercicios que, al mismo tiempo, le ayudaba a encontrar sentidos y comprender mejor su contexto.

Un propósito fundamental del cuerpo de docentes líderes era transformar las concepciones acerca de los museos como lugares archivadores de objetos, es decir, desarrollar una conciencia museal, como la llaman, y volverlos un contexto dinámico y participativo.

Cambiar la concepción de museo como el guardador de objetos. Entonces, se le da otra connotación, que se esté... dinamizando a través de los proyectos de aula y que el mismo niño sea constructor de ese museo, que no sea que venga a mirar en una clase todo lo que hay allí exhibido y no sabemos si le queda algo, sino que él mismo haga parte de ese proceso y pueda quedársele. Como fundamentar esos conocimientos, cimentarlos más... Esa conciencia museal permite que el estudiante tenga esos conocimientos más fijos y tenga amor por lo propio. (D.1, comunicación personal, marzo de 2014) 
Esta nueva concepción con sentido pedagógico, parafraseando a Tasky (2008), permitía cuestionar los orígenes de la identidad individual y colectiva, pues los procesos que la configuraban, representados en los objetos que se exhibían, no eran valores ni tradiciones estáticas que se mostraban como paquetes terminados. De este modo, era el patrimonio que se construía bajo la luz del presente. Asimismo, como lo señalaba la museología actual, este lugar adquiría el significado que sus creadores y visitantes le asignaban, que, en el caso de Historichoquiando, era reivindicativo, pues su objetivo era el rescate y la valoración de la historia, la cultura y la identidad local y regional.

En este sentido, Historichoquiando, como su nombre lo indicaba, fue una oportunidad para la población del Chocó de hacer y contar su propia historia, la cual era excluida y marginada de los grandes relatos del país. Los resultados de la investigación hicieron evidente una experiencia educativa de tipo político, que buscaba el reconocimiento de la afrocolombianidad, relegada de las narraciones en los textos escolares oficiales. De la misma manera, reclamaba no solo mayor atención a su realidad, sino a su experiencia, como el reflejo de una construcción social y cultural específica.

\section{Los Derechos Humanos: una experiencia para la formación ciudadana y la convivencia escolar}

La segunda experiencia significativa, $A$ Crecer con Derechos, problematizaba la relación entre Derechos humanos y formación ciudadana, y su relevancia para el contexto, con el fin de establecer vínculos con las prácticas formativas. Dentro de sus objetivos, los Derechos Humanos eran concebidos como un punto de partida de la intervención pedagógica para transformar las relaciones de la comunidad educativa desde el sentir, el pensar y el actuar, de modo que eran la base para la intervención pedagógica en los asuntos de convivencia.

\section{Los Derechos Humanos, una opción para transformar la realidad escolar y formar en ciudadanía.}

A partir de la preocupación del profesorado de las áreas de Ciencias Sociales, Educación Artística y Educación Física para dar alternativas a las problemáticas que permeaban el contexto de su comunidad educativa, se propuso la creación de espacios dirigidos hacia la formación ciudadana, para lo que se tomó como plataforma la formación política en Derechos Humanos. Como consecuencia, pensarse políticamente fue un objetivo que trascendió el aula de clase para convertirse en la razón de ser de la formación ciudadana.

Entendimos que un proyecto de derechos humanos en la escuela es una propuesta de formación política como tal, que desborda los límites que a veces impone lo pedagógico. Entonces, nosotros lo concebimos desde sus inicios como un proyecto de formación política, ya que la educación la entendemos como un acto político y afectivo que da sentido a los derechos humanos en la escuela (D.1, comunicación personal, 6 y 7 de marzo de 2014).

De esta manera, los elementos políticos brindaron la posibilidad de pensar la escuela como escenario para la participación y la toma de decisiones. En este sentido, la formación política del estudiantado tomó fuerza en la medida en que se abrieron a la posibilidad de reflexionar en su ejercicio participativo y no representativo. Es decir, su voz y voto contaron dentro del espacio escolar y fomentaron una cultura democrática en el marco del derecho a la participación. En esta línea, según Jares (2006), la formación política es

...un requisito para el ejercicio efectivo de los derechos humanos y de las libertades fundamentales, por lo tanto, conceder una atención particular a la educación en derechos humanos y en democracia es una condición esencial para el pleno desarrollo de la justicia (p. 58). 
Con respecto a la formación en derechos, esta se abordó estratégicamente a partir del cuerpo y según a autores como Planella (2005), quien cuestionaba cómo la relación entre el cuerpo y la pedagogía se había fundamentado en su dimensión física, mientras desconocía su dimensión simbólica, ante lo que el autor plantea no situar al cuerpo en un territorio al margen de la persona, sino en el espacio central de la escenografía. Además, de acuerdo con Détrez (2002), el cuerpo se construye socialmente y posee una serie de connotaciones simbólicas que permiten hablar de él como palabra, mensaje y hermenéutica. Bajo este horizonte de sentido se formuló el proyecto $A$ crecer con derechos, a partir de un principio de transformación curricular nombrado ;Sin cuerpo no hay derechos! y cuatro fundamentos derivados por el grupo de docentes, los cuales se consideraron pilares en la configuración de la propuesta y en las actividades llevadas a cabo. Estos fueron:

- ;Sin cuerpo no hay derechos!: El cuerpo se consideraba como territorio de lo humano y desde allí se generaba una relación consigo mismo y con el otro. De este modo, se vinculaban los Derechos Humanos con los ideales de la construcción corporal para generar un respeto por la vida de sí mismo y de otros.

- ¡Los derechos no se otorgan, se exigen!: Educarse en Derechos Humanos no solo implicaba conocer qué eran, de dónde provenían y por qué eran importantes. En esta medida, no era suficiente con exigirse a sí mismo su conocimiento, sino que se requerían las exigencias del y para el otro, su reconocimiento, defensa y promoción.

· iLos derechos no se aprenden, se viven!: Este principio partía de problematizar que el reconocimiento de los Derechos Humanos en la escuela no era tarea fácil, debido a que no existía una tradición fuerte y una vivencia de estos en la cultura colombiana. Por lo tanto, se exploraron metodologías que garantizaran su vivencia, promoción y defensa, particularmente por medio de una pedagogía experiencial y lúdica.

- ¡No hay derechos sin los otros!: Como resultado de estos imaginarios surgió la preocupación sobre dónde quedaban los deberes. Este principio pretendía responder esta interrogante, al considerar que los derechos eran una construcción histórica y que al reconocerlos en cada ser humano se debía admitir la responsabilidad frente a su defensa, respeto y promoción, tanto de los propios como los de los demás.

- Los derechos humanos como eje articulador para la integración curricular: A partir de las necesidades del contexto se propuso la creación de una malla curricular que incluyera los Derechos Humanos e impulsara un nuevo modelo de formación, centrado en el sujeto y la forma en que se construye con otro a partir de su realidad y sus proyecciones de vida, dado que la institución educativa estaba siendo afectada por bandas delincuenciales, microtráfico de estupefacientes y pandillas juveniles.

Con base en lo anterior, la construcción de la malla curricular cubrió todos los grados, ciclos y áreas de la institución; además, permeó las necesidades de la comunidad frente a la formación en valores. Sin embargo, esta se transformó a partir de la implementación de la experiencia, ya que cada una de las actividades y los enfoques planteados exigían una valoración para determinar en qué se podía mejorar para cumplir los objetivos propuestos, no solo en términos metodológicos, sino, también, en la convivencia escolar. Lograr esta transformación curricular fue un hecho que cambió la cultura de la institución en términos de Derechos Humanos, convivencia y formación ciudadana.

Como institución, hemos mejorado porque le hemos dedicado nueve años de trabajo serio a la convivencia y tenemos un proyecto interesante junto con el comité de convivencia institucional que maneja y lidera a través de un sistema integrado de gestión, donde todo lo hacemos de manera integral y general para la institución. Por ello la relación en derechos humanos, formación ciudadana y convivencia es fundamental para nosotros. (P.1, comunicación personal, 6 y 7 de marzo de 2014)

Así, esta nueva forma de pensar el currículo fue una oportunidad para guiar los procesos educativos centrados en el sujeto, su sentir, pensar y actuar, y resolver constructivamente los conflictos del contexto que permeaban a la institución educativa. 


\section{Formación ciudadana a partir de la pedagogía urbana}

La tercera experiencia se analizó desde el enfoque de Pedagogía Urbana[2], que reconoce a las estudiantes como ciudadanas con un rol y una función que cumplir en la ciudad a partir de su participación, y a esta última como un escenario de aprendizaje y formación. Por esta razón, era necesario que cada estudiante, desde temprana edad, se relacionara con su entorno social, comunitario y educativo. La experiencia ha logrado desarrollar varios proyectos formativos desde el enfoque de la pedagogía urbana, entre los cuales se resaltan:

- El estudio de la localidad: un encuentro de las estudiantes con su realidad en el año 1996.

- Conocimiento de la ciudad de Barranquilla a partir del transporte urbano en el año 2000.

- Marcando pasos en nuestra ciudad de Barranquilla en el 2005.

- Explorando el espacio público y la cultura ciudadana en la ciudad de Barranquilla en el 2010.

- La historia de mi familia y de mi barrio a través de la indagación social escolar en el 2014. (D.1, comunicación personal, 6 y 7 de marzo de 2014)

\section{La ciudad como escenario de formación ciudadana.}

De acuerdo con los planteamientos del docente líder, existía un gran abismo entre la escuela y la ciudad. Así, En la escuela circulan saberes alejados y desarticulados totalmente de la realidad del entorno social inmediato y mediato del estudiantado. Es poco lo que la escuela hace pedagógicamente para que estos conozcan y reconozcan su barrio, su localidad, su ciudad, departamento, región y país (Cortina, 2010, p.4).

En ese sentido, pensar en la institución y la ciudad era considerar y vivir la escuela fuera de sus instalaciones o infraestructura física, sin limitarse estrictamente al cumplimento de los horarios establecidos por la normatividad y pensando en los aportes y relaciones tanto entre la institución y el contexto como con la cultura y la ciudadanía. Por eso, había un gran reto: contribuir hacia y para la formación ciudadana, generando capacidades, tanto en las estudiantes como en los ciudadanos, para afrontar la vida a partir de las realidades del escenario urbano.

Este proyecto ha rescatado la historia que no conocía. En algunas ocasiones hay colegios que desconocen todo el conocimiento que puede aportar el contexto que se encuentra alrededor. Y para este proyecto se ha tomado la tarea de descubrir la historia que guarda, a través de la indagación social, y esta información la posee personas que se dedican al trabajo como zapatería, vendedores de comida, el tendero, entre otros, que conocen elementos importantes del cómo nació y quiénes fundaron los barrios aledaños. De esta forma, al reunir tantas voces, se va construyendo la historia de mi barrio, mi gente, etc. (P.1, comunicación personal, 6 y 7 de marzo de 2014)

Según lo anterior, la experiencia planteaba que, al indagar la ciudad, el estudiantado evidenciaría diversos temas relacionados con su formación ciudadana, como la participación, la toma de decisiones, la cultura ciudadana y el desarrollo moral. Este último fue un factor decisivo para desarrollar la sensibilidad moral que anteponía el bien público sobre el particular. Así, se posibilitaba una postura crítica en la vida política y social que aparta la autonomía en las decisiones que debían afrontar.

\section{La investigación no es para sabios, sino para aquellos que quieren aportar y construir conocimiento a partir de su realidad.}

Metodológicamente la experiencia se desarrolló por medio de la práctica investigativa, se partió de lo que se tenía, podía y quería hacer desde un fin común: construir y aportar conocimiento a partir de las realidades más inmediatas. Esta experiencia se centró en hacer del espacio público un elemento fundamental para la enseñanza y el aprendizaje de las Ciencias Sociales a partir de la indagación, consulta y observación de los 
escenarios que ofrecía la ciudad, como parques, museos, teatros, paraderos de buses, calles principales, entre otros.

Entonces, la cultura ciudadana parte de conocer la ciudad y parte, también, de lo macro, calles, carreras, lugares que son de la ciudad y que seguramente las niñas no conocían y tiene que ver concretamente con conocer la ciudad, visitándola, haciendo encuestas, registrando lo que observan, tomando fotos y aprendiendo del adecuado comportamiento en cada uno de esos espacios. (P.2, comunicación personal, 6 y 7 de marzo de 2014)

De esta forma, se motivaba a que las estudiantes conocieran y reconocieran las reglas y leyes que se presentaban en cada espacio. En esta línea, de acuerdo con Páramo (2009), las estudiantes conocieron los códigos y las normas de regulación del transporte público e interpretaron la lógica de la planificación urbana que este conllevaba. También, realizaron los planos de los barrios, crearon un archivo fotográfico y de videos de la ciudad, visitaron los lugares de la memoria y agenciaron trabajos de responsabilidad social compartida.

Para concluir, cabe mencionar que, durante todos estos años de implementación, la experiencia se preocupó por sistematizar, registrar y difundir sus resultados a nivel local, nacional e internacional por medio de la participación en espacios académicos. Entonces, se evidenció que era posible concebir la institución educativa como ciudad y viceversa.

\section{DiscuSión Y CONCLUSIONES}

Si bien las experiencias partían de contextos específicos - ya que la primera trabajaba la ciudadanía a partir de referentes identitarios y culturales; la segunda, a partir de los Derechos Humanos; y la tercera, desde la participación en la ciudad - las intencionalidades del profesorado se alineaban en la búsqueda de una formación política de sus estudiantes como ciudadanos, ciudadanas y sujetos políticos. Sin embargo, es necesario un mayor desarrollo de capacidades para el debate argumentado, un conocimiento más documentado de la realidad nacional e internacional y mayores habilidades para agenciar procesos organizativos de carácter colectivo, que son ámbitos fundamentales para la formación ciudadana.

Además, se observó una mayor integración curricular que apunta hacia la interdisciplinariedad, aspecto fundamental para garantizar el desarrollo de capacidades ciudadanas en el estudiantado y un mayor énfasis del profesorado en el desarrollo de conocimientos y emociones políticas dentro de sus prácticas educativas.

Por otro lado, los ámbitos de la formación ciudadana trascendieron el fortalecimiento de la convivencia, el gobierno y la mediación del conflicto escolar, al relacionarse con la formación política del estudiantado, el reconocimiento de la historia local y la valoración de la identidad como referente de pertenencia y la ciudad como escenario de participación ciudadana. Los hallazgos confirman procesos formativos que transitan de una educación cívica con enfoque en la civilidad (educación sobre ciudadanía) hacia una formación ciudadana (educación por medio de la ciudadanía y para la ciudadanía), donde se promueve la participación del estudiantado y la toma de decisiones en el escenario escolar, lo que lo prepara para participar en la esfera pública. En este contexto, los aportes derivados de la investigación se sitúan en el modelo de formación ciudadana que pretende desarrollar la autonomía como emancipación individual; esto para formar estudiantes que aprendan a ejercer sus derechos, cumplir con sus deberes y participar en la transformación de sus contextos.

Por otra parte, según las dimensiones más abordadas en formación ciudadana, el aspecto emocional primó como parte constitutiva del sujeto, que se configuró como un ser sentipensante, donde la empatía es fundamental para el ejercicio ciudadano. Tal como lo señalaba Cortina (2010), las personas no solo son razón, son, también, corazón y, para alcanzar el ideal de sociedad, no basta solamente con los argumentos de la razón, sino que es necesario cultivar los sentimientos, ya que son los que les permiten identificarse y vincularse con los otras. Por consiguiente, esta dimensión tenía la misma importancia que la cognoscitiva, en contraste con la relevancia que tuvo en los procesos formativos hace nueve años. 
En relación con los procesos de aprendizaje privilegiados, se encontró que las experiencias promovían aprendizajes significativos basados en problemáticas del entorno y culturalmente relevantes, de modo que las pautas de relación y el lenguaje hacían posible el reconocimiento del otro, la construcción de acuerdos y la formulación de proyectos para el contexto local. Así, la observación, el análisis y la problematización eran los procesos de aprendizaje que se privilegiaban.

Un hallazgo importante fue que las experiencias promovían procesos democráticos, pues, al rescatar y valorar los saberes tradicionales comunitarios y ponerlos a dialogar con los saberes académicos, se dio un intercambio que generó la construcción compartida de conocimientos. En este sentido, se evidenció que el saber académico requería de la cultura local para adquirir sentido y pertinencia, donde el profesorado jugó un gran papel para configurar el currículo desde su autonomía y la mediación entre el contexto cultural y el estudiantado.

De esta manera, lo que se enseñaba y aprendía en la escuela partía y se reflejaba en lo inmediato; así convertía los conocimientos en aprendizajes significativos que se aplicaban a la vida cotidiana.

Otro hallazgo importante fue la evaluación, pues las experiencias adelantaban procesos evaluativos sistemáticos que lograron evidenciar cambios en el estudiantado y en los contextos de su accionar. De hecho, lo encontrado en la experiencia Historichoquiando fue una evidencia de que las acciones pedagógicas no se limitaban al contexto escolar, sino que adquirían significados de tipo cultural, social y político. Ello se observaba en nuevos relatos históricos, nacidos de la necesidad de reconocimiento que tenían sectores sociales excluidos de las grandes historias.

De acuerdo con los planteamientos anteriores, fue posible identificar un eje articulador de los ámbitos del saber de la formación ciudadana en las experiencias, en este caso fue la formación política del estudiantado, en torno a la cual se desplegaron los proyectos con diversidad temática y de abordaje metodológico según los contextos. En este sentido, las perspectivas metodológicas privilegiadas fueron la investigativa, la vivencial y la histórico-cultural, las cuales acudieron a la vida cotidiana, los problemas del entorno y las experiencias personales del estudiantado. Como referentes pedagógicos sobresalía: pedagogía urbana y pedagogía experiencial, las cuales otorgaban sentido a las prácticas educativas del profesorado para la formación ciudadana.

Con relación a las intencionalidades de formación en lo social y político planteadas por el profesorado, este decidió ir más allá de los límites que imponían las competencias ciudadanas, pues le apostaron a una formación política del estudiantado en el escenario escolar. Se concebía la educación como acto político y afectivo que daba sentido a los derechos en la vida escolar, familiar y comunitaria. Por tanto, pensarse políticamente fue un objetivo que trascendió el aula de clase y las paredes de la escuela, para convertirse en la razón de ser de la formación ciudadana al salirse de los estándares y lo preestablecido de una educación perfeccionista, para así formar ciudadanos y ciudadanas que participen en la construcción de un proyecto común de humanidad.

Por otro lado, las resignificaciones elaboradas por el profesorado en materia de formación ciudadana, remarcan un interés por la formación política de sus estudiantes, su construcción en la esfera de lo público, pero, también, desde la intimidad del sujeto (su sentir, pensar y actuar), en la cual la honestidad de los sentimientos morales y las emociones políticas se encuentran y fortalecen.

En consideración de las limitaciones de este estudio, se debe señalar que, aunque hay importantes avances en términos de la sistematización de la práctica y las comprensiones que el profesorado construyó en torno a la formación ciudadana, todavía falta una mayor vinculación de los colectivos docentes para intercambiar experiencias educativas en las redes y organizaciones del profesorado, y desarrollar más investigación en torno a la formación ciudadana. Por otro lado, persiste la falta de una síntesis teórica alrededor de los conceptos ciudadanía y formación ciudadana, lo que pone en evidencia que todavía falta transitar mucho camino.

En este orden de ideas, formar para la ciudadanía supone apostarle a un modelo pedagógico en el que el estudiantado aprenda a practicar lo que significa ser ciudadano y ciudadana desde los primeros años de 
escolaridad. Además, implica reflexionar sobre lo que esto conlleva mientras aprende a construir un modo de vida comunitario. Para tal fin se recomienda gestionar en la escuela semilleros de investigación orientados a la producción de conocimiento sobre los problemas nacionales, que generen prácticas ciudadanas que fortalezcan el vínculo escuela-comunidad, el interés por el bien común, la participación, la toma de decisiones y la formación como ciudadanos, ciudadanas y actores políticos. Asimismo, fortalecer las redes del profesorado para intercambiar experiencias y conocimientos que enriquezcan la discusión y el debate en la formación ciudadana.

El proceso de globalización que se está viviendo, la discriminación de género, los movimientos migratorios, el desarrollo sustentable y las transformaciones sociales y tecnológicas plantean nuevos retos a la formación ciudadana, los cuales se deben asumir con más fuerza en los deberes hacia la humanidad, y con menos exigencias en los derechos para consigo mismo.

\section{Referencias Bibliográficas}

Abowitz, K. K.y Harnish, J. (2006). Contemporary Discourses of Citizenship. Review of Educational Research, 76(4), 653-690. Recuperado de https://journals.sagepub.com/doi/pdf/10.3102/00346543076004653

Avendaño-Castro, W., Paz-Montes, L. y Parada-Trujillo, A. (2016). Construcción de ciudadanía: un modelo para su desarrollo en la escuela. El Ágora USB, 16(2), 479-492. doi: https://doi.org/10.21500/16578031.2444

Bickmore, K., Salehin-Kaderi, A. y Guerra-Sua, Á. (2017). Creating capacities for peacebuilding citizenship: history and social studies curricula in Bangladesh, Canada, Colombia, and México. Journal of Peace Education, 14(3), 282-309. doi: https://doi.org/10.1080/17400201.2017.1365698

Caicedo-Caviedes, L. D. (2015). Propuesta curricular para el desarrollo de las competencias ciudadanas desde la Educación Física (Tesis de pregrado). Recuperado de https://bit.ly/399xYwo

Carretero, M. y Montanero, M. (2008). Enseñanza y aprendizaje de la Historia: aspectos cognitivos y culturales. Revista Cultura y educación, 20(2), 133-142. Recuperado de https://bit.ly/31hInC0

Chaux, E. (2012). Educación y convivencia escolar. Bogotá: Taurus

Chaux, E., Lleras, J. y Velásquez, A. M. (2004). Competencias Ciudadanas: De los Estándares al Aula. Una propuesta de integración a las áreas académicas. Recuperado de https://bit.ly/3rozeC6

CINDE. (2010). Informe descriptivo y analitico de programas de formación en competencias ciudadanas. Manizales: CINDE-CERLALC.

Conejo-Rodri\#guez, P. A. (2012). El valor formativo de la mu\#sica para la educacio\#n en valores. DEDiCA. REVISTA DE EDUCAC\#A\#O E HUMANIDADES, 2, 263-278. Recuperado de https://dialnet.unirioja.es/servlet/artic ulo?codigo $=3825651$

Cortina, A. (2010). Ética de la razón cordial: educar en la ciudadania en el siglo XXI. España: Ediciones Nobel.

de Oliveira Andreotti, V. (2011). (Towards) decoloniality and diversality in global citizenship education. Globalisation, Societies and Education, 9(3-4), 381-397. doi: https://doi.org/10.1080/14767724.2011.605323

de Oliveira Andreotti, V. y Pashby, K. (2013). Digital Democracy and Global Citizenship Education: Mutually Compatible or Mutually Complicit? The Educational Forum, 77(4), 422-437. doi: https://doi.org/10.1080/0 0131725.2013 .822043

Détrez, C. (2002). La construction sociale du corps. París: Seuil.

Eidhof, B. B., ten Dam, G. T., Dijkstra, A. B., y van de Werfhorst, H. G. (2016). Consensus and contested citizenship education goals in Western Europe. Education, Citizenship and Social Justice, 11(2), 114-129. doi: https://do i.org/10.1177/1746197915626084

Garzón-Guerra, E. y Acuña-Beltrán, L. F. (2016). Integración de los proyectos transversales al currículo: una propuesta para enseñar ciudadanía en ciclo inicial. Revista Actualidades Investigativas en Educación, 16(3), 1-26. doi: http ://dx.doi.org/10.15517/aie.v16i3.26065

Gergen, K. J. (1982). Hacia la transformación social del conocimiento. Nueva York: Springer. 
Gergen, K. J. (1999). An invitation to social construction. Londres: Sage.

Gimeno-Sacristán, J. (2001). Educar y Convivir en la Cultura Global. Madrid: Editorial Morata.

González-Valencia, J. A., Posada-López, R. del P. y Valencia-Calvo, C. H. (2012). La enseñanza para la participación en el currículo de las Ciencias Sociales en la educación obligatoria en Colombia. En: N. de Alba-Férnández, F. F. García-Pérez y A. Santisteban-Fernández (Coords), Educar para la participación ciudadana en la enseñanza de las ciencias sociales (125-130). Sevilla: Asociacio\#n Universitaria de Profesorado de Dida\#ctica de las Ciencias Sociales-Díada editora, S. L.

Hoyos-Vásquez, G., Serna-Arango, J. y Gutiérrez-Ruiz, E. F. (2007). Borradores para una filosofía de la educación. Bogotá: Siglo del Hombre.

Jares, X. R. (2006). Educación en Derechos Humanos. Madrid: Editorial Popular.

León-Muñoz, S. y López-Takegami, J. (2015). Formar ciudadanos desde el pre-escolar. Educación y Educadores, 18(2). Recuperado de https://bit.ly/31gmliW

López, M. B., Arán-Filippetti, V., y Richaud, M. C. (2013). Empatía: desde la percepción automática hasta los procesos controlados. Avances En Psicología Latinoamericana, 32(1), 37-51. doi: https://doi.org/10.12804/apl32.1.201 4.03

Marchesi, Á. (2011). Preámbulo. En: B. Toro y A. Tallone (Coords.), Educación, valores y ciudadanía. Madrid: OEIFundación SM.

Marshall, T. H. (1950). Citizenship and Social Class, and Other Essays. Cambridge: The Syndics of the Cambridge University Press.

Ministerio de Educación Nacional (MEN). (2003). Estándares básicos de competencias ciudadanas. Educación Básica y Media. Bogotá: MEN-Ascofade.

Ministerio de Educación Nacional (MEN). (2004). Estándares básicos en competencias ciudadanas: formar para la ciudadanía si es posible. Bogotá: MEN.

Ministerio de Educación Nacional (MEN). (2010). Las rutas del saber hacer: Experiencias Significativas que transforman la vida escolar. Orientaciones para autores de experiencias y establecimientos educativos. Bogotá: MEN Recuperado de https://aprende.colombiaaprende.edu.co/ckfinder/userfiles/files/guia_37.pdf

Ministerio de Educación Nacional (MEN) y Comitato Internazionale per lo Sviluppo dei Popoli (CISP). (2012). Las competencias ciudadanas en el territorio y la comunidad. Documento inédito.

Muñoz-Labraña, C. y Torres-Durán, B. (2014). Civics Education at Schools: Problems and Challenges. Revista Electrónica Educare, 18(2), 233-245. doi: https://doi.org/10.15359/ree.18-2.12

Náñez-Rodríguez, J.J.y Castro-Turriago, H. M. (2016). Educación artística y formación ciudadana: espacio para forjar la sensibilidad en la Corporación Colegio San Bonifacio, de Ibagué, Colombia. Entramado, 12(2), 154-165. doi: https://dx.doi.org/10.18041/entramado.2016v12n2.24221

Páramo, P. (2009). Metacontigencias y cambios de prácticas culturales en el espacio. Bogotá: Instituto de Investigación y Proyectos Especiales-Universidad Piloto de Colombia.

Pinilla-Díaz, A. V. y Torres-Azocar, J. C. (2006). De la educación para la democracia a la formación ciudadana: una década de incertidumbres. Bogotá: Universidad Pedagógica Nacional-IDEP.

Planella, J. (2005). Pedagogía y hermenéutica del cuerpo simbólico. Revista de Educación, (336), 189-201. Recuperado de http://femrecerca.cat/sites/default/files/jordi_planella/files/re336_11-1.pdf

Pontificia Universidad Católica de Chile. (2010). Informe regional 2010. Evaluación de Programas Exitosos de Formación Ciudadana. Bogotá: SREDECC-CERLALC.

Rodríguez, G. (2010). Proyectos pedagógicos en construcción de ciudadanía. Bogotá, Colombia: Ministerio de Educación Nacional MEN - Comitato Internazionale per lo Sviluppo dei Popoli CISP.

Saldarriaga-Vélez, J. A. (2016). Las escuelas críticas: Entre la socialización política y los procesos de subjetivación. RLCSNJ, 14(2). Recuperado de https://bit.ly/2QyfoHP

Selwyn, N. (2004). Literature Review in Citizenship, Technology and Learning. Bristol: Futurelab. 
Silva, D. A. y Tapia-Zúñiga, A. (2015). Desafios de la formación ciudadana en la era digital. Valparaíso: Biblioteca del Congreso-Fondo de Cooperación Chile-México. Recuperado de https://bit.ly/2Pog6qK

Sosa-Cabrera, S. (2002). La génesis y el desarrollo del cambio estratégico: un enfoque dinámico basado en el momentum organizativo (tesis doctoral). Recuperado de http://www.eumed.net/tesis/2006/ssc/

Tasky, A. (2008). Usos del pasado, patrimonio, identidad y museos en discusión. Revista Clío \& Asociados, (12), 29-55. Recuperado de https://bit.ly/2Pmbrp8

Vargas-Torres, M. R. (2010). Towards a Discourse for Criticism in Language Teaching: Analysis of Sociocultural Representations in Mass Media. Profile: Issues in Teachers'ProfessionalDevelopment, 12(2), 179-190. Recuperado de https://revistas.unal.edu.co/index.php/profile/article/view/17692

Zapata-Barrero, R. (2001). Ciudadanía, democracia y pluralismo cultural: hacia un nuevo contrato social. Barcelona: Anthropos.

\section{Notas}

[1] Esta mirada histórica de la ciudadanía fue compartida por el politólogo Neftalí David Suarez de la Facultad de Ciencias Políticas de la Pontificia Universidad Javeriana.

[2] La pedagogía urbana nació en Colombia a partir del movimiento Ciudad educadora en los años ochenta. Lo relevante de esta pedagogía fue el planteamiento de la transversalidad de temas para la formación ciudadana, como la educación ambiental, la historia y la identidad municipal, la promoción cultural y artística, el fortalecimiento y el uso de los espacios públicos, la convivencia y la participación ciudadana.

\section{INFORMACIÓN ADICIONAL}

Cómo citar: Lara Salcedo, L.M. (2021). Sentidos y significados de la formación ciudadana en tres escuelas colombianas Revista Educación, 45(2). Recuperado de http://doi.org/10.15517/revedu.v45i1.42799 